\title{
La familia Rhodomellaceae (Ceramiales, Rhodophyta) en la costa del estado de Michoacán, México.
}

\author{
Abel Sentíes G., Francisco F. Pedroche y Kurt M. Dreckmann ${ }^{1}$
}

\begin{abstract}
RESUMEN. En este trabajo se caracterizan a los representantes mesomareales de la familia Rhodomelaceae, presentes en las costas michoacanas. Se proponen listas de caracteres y una ponderación preliminar para ser utilizados en la descripción de las especies de los géneros de dicha familia. Se presentan claves genéricas y específicas. Se hace un análisis de la distribución a lo largo del litoral michoacano y se discuten algunas de las posibles causas para la presencia y ausencia de las especies, tomando en cuenta fundamentalmente los habitats que ocupan. Se enfatiza en diferencias morfo-anatómicas entre lo reportado y lo encontrado en los especímenes, ampliando en algunos casos sus intervalos de variación. La flora que se analizó, consta de catorce especies distribuídas en cinco géneros, destacando seis nuevos registros para México en sus costas tropicales: Polysiphonia confusa, P.scopulorum, Chondria arcuata, C. dasyphylla, Laurencia clarionensis y L. hancockii.
\end{abstract}

ABSTRACT. In this paper, the Rhodomelaceae family intertidal representants are characterized in the coasts of Michoacan. A list of taxonomic characters and a preliminary weight of them are proposed for use in the determination and diagnosis of the species in each genus of the family. The elaboration of a sinoptic key for the genera and species investigated was carried out. An analysis about the distribution of the species through the litoral of Michoacan was made and some of the causes for the presence and absence of them are discussed, considering mainly the habitat where they grow. An emphasis was made in morphologic and anatomic variation between the previous records and our material, and in some cases, a new range of variation is giver. The analized flora is composed of fourteen species, distributed in five genus, pointing out six new records for Mexico in its tropical coasts: Polysiphonia corfitisa, P. scopulorum, Chondria arcuata, C. dasyphylla, Laurencia clarionensis y L. hancockii.

${ }^{1}$ Laboratorio de Ficología, Departamento de Hidrobiología, División C.B.S. Universidad Autónoma Metropolitana - Iztapalapa. Apdo. Postal 55-535; 09340 México, D.F.

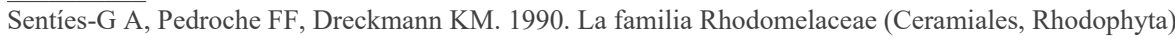
en la costa del estado de Michoacán, México. Boletín de la Sociedad Botánica de México 50: 89-120. 
En la ficología mexicana los trabajos de índole florística enfrentan problemas entre los que podemos mencionar la dualidad que se presenta entre terminar o más bien actualizar el inventario del recurso algal de la manera prospectiva, entendiendose ésta como la idea general o preliminar sobre la presencia-ausencia de los diversos representantes de las divisiones algales, en localidades o regiones determinadas, o bien la elaboración de estudios más detallados o profundos sobre tal o cual taxon, lo que necesariamente conlleva a retomar toda la información existente sobre el grupo en cuestión y valorar la fidelidad de los registros y la certeza de la determinación taxonómica. Máxime cuando se trata de grupos como las algas en los que las revisiones son escasas.

Esto nos lleva a enfrentar como ya se ha mencionado anteriormente la lección de seguir empleando los métodos convencionales de determinación o proponer alternativas que puedan de alguna manera agilizar, facilitar y hacer homogéneo el trabajo taxonómico en ficología (Flores-Pedroche, 1981).

En este estudio se aborda a la familia Rhodomelaceae presente en la costa de Michoacán con la finalidad de resaltar el análisis de los caracteres que han sido utilizados en diferentes trabajos para la delimitación de los géneros y de las especies en cada uno de ellos, proponiendose una lista de atributos que podrían ser empleados para la caracterización de las entidades y de esta manera permitir la comparación con otras entidades presentes en espacios y tiempos diferentes. De esta forma las posibilidades de evidenciar variación y estabilidad de los atributos y la interpretación de ellos, podría llevarnos a realizar en un mediano plazo estudios ya no prospectivos sino profundos y con elementos de valoración con la consecuente propuesta sobre la unificación de criterios de análisis.

La familia Rhodomelaceae Harvey, agrupa cerca de 125 géneros y aproximadamente 600 - 625 especies, considerándose la familia más grande de la división Rhodophyta. Los caracteres intrínsecos de la familia según Scagel (1953) son: presencia común de tricoblastos e início del pericarpo antes de la fertilización.

La posición filogenética de la familia se considera como la más evolucionada dentro del orden, por la fecundación directa de la célula auxiliar y su temprana formación, además del alto grado de desarrollo del pericarpo y de las células protectoras Scagel, (1953).

La corriente más actual referente a la sistemática, es la postulada por Scagel (1953), quien divide a la familia en 14 subfamilias, sectorizandolas de acuerdo a criterios de tipo evolutivo y de características morfo-anatómicas. Sin embargo, a estas subfamilias se han agregado dos más: Brongniartelleae por Parsons en 1975 y Rhodolachncae por Womersley y Bailey en 1970 (Kraft, 1981) usando los mismos criterios que Scagel, quedando conformada la familia Rhodomelaceae por 16 grupos o subfamilias.

Dentro de los trabajos más relevantes que abordan a la familia como un todo están Scagel (1953) y Homersand (1963) estudios con enfoque morfológico, de caracterización 
con ejemplos de ciertos géneros y especies, mencionando datos referentes a la evolución de la familia, su posición filogenética y su relación con otras familias del orden.

Con respecto a estudios florísticos, que aportan información de la familia pero integrada con la de otros grupos algales están: Taylor (1945); Dawson (1963); Joly (1967); Oliveira (1967) y Abbott y Hollenberg (1976). Asimismo, existen estudios referentes a géneros representativos de la familia, como ejemplo citaremos los trabajos de Hollenberg (1961, 1968 y 1968a); Taylor (1966); Ardré (1967); Saito (1967); Saito y Womersley (1974); Hollenberg y Norris (1977) y Womersley (1979).

En relación con el área (costas del Edo. de Michoacán), destacan los trabajos de tipo florístico: Martinell (1983) y (1986) y Pedroche et al(1984). Esta escasez de investigación en el estado, se debió principalmente a la falta de accesibilidad a su costa. Posterior a ellos cabe mencionar, los trabajos de Sentíes (1985); Correa (1986) y Dreckmann (1987).

\section{ÁREA DE ESTUDIO}

Después de haber llevado a cabo el recorrido de las costas del estado se delimitaron tres grandes áreas; la primera de la boca del Río Coahuayana a la base de la península de San Juan Lima; la segunda desde éste último punto hasta Las Peñas y la tercera, desde Las Peñas hasta la desembocadura del Río Balsas.

La primera y tercera secciones están constituídas por largas playas cuyas arenas representan un sustrato poco adecuado para el desarrollo de algas bénticas. Sin embargo, existen puntos rocosos dispersos que pueden representar lugares importantes de muestreo (Pedroche, et al., 1984).

La porción intermedia y más extensa $(145 \mathrm{~km}$ ) forma un litoral con pequeños valles y playas intercaladas, en la mayor parte de este tramo las rocas ígneas descienden de las altas colinas para terminar abruptamente en el mar.

En esta última zona se ubicaron 6 grandes lugares de recolección: San Telmo, Coalcoman, Maruata, Pichilinguillo, Mexcalhuacan y Las Peñas. De ellos se eligieron 3 para este estudio (fig. 1): San Telmo, que es un localidad ubicada a $36 \mathrm{~km}$ de Tecomán, Col. y con acceso por la carretera Fed.-200 Playa Azul-Tecomán en el km 255.5, por el poblado Ojo de Agua; es una gran punta rocosa, limitada en su extremo norocste por una larga playa arenosa y por el sureste, un gran grupo de rocas impide establecer un límite preciso. La siguiente localidad Pichilinguillo, se encuentra en el km 100 de la carretera Fed.-200; es una bahía limitada por dos puntas rocosas, dentro de la cual se localizan tres pequeñas playas arenosas separadas por salientes rocosas. Como última localidad se eligió a Caletilla de Mexcalhuacan, la cual esta aproximadamente a $41 \mathrm{~km}$ del poblado Caleta de Ocampo; es una pequeña caleta, carece de arena, se delimita por una punta y por una plataforma rocosas, también existe una porción de cantos rodados que forman una pequeña playa. 


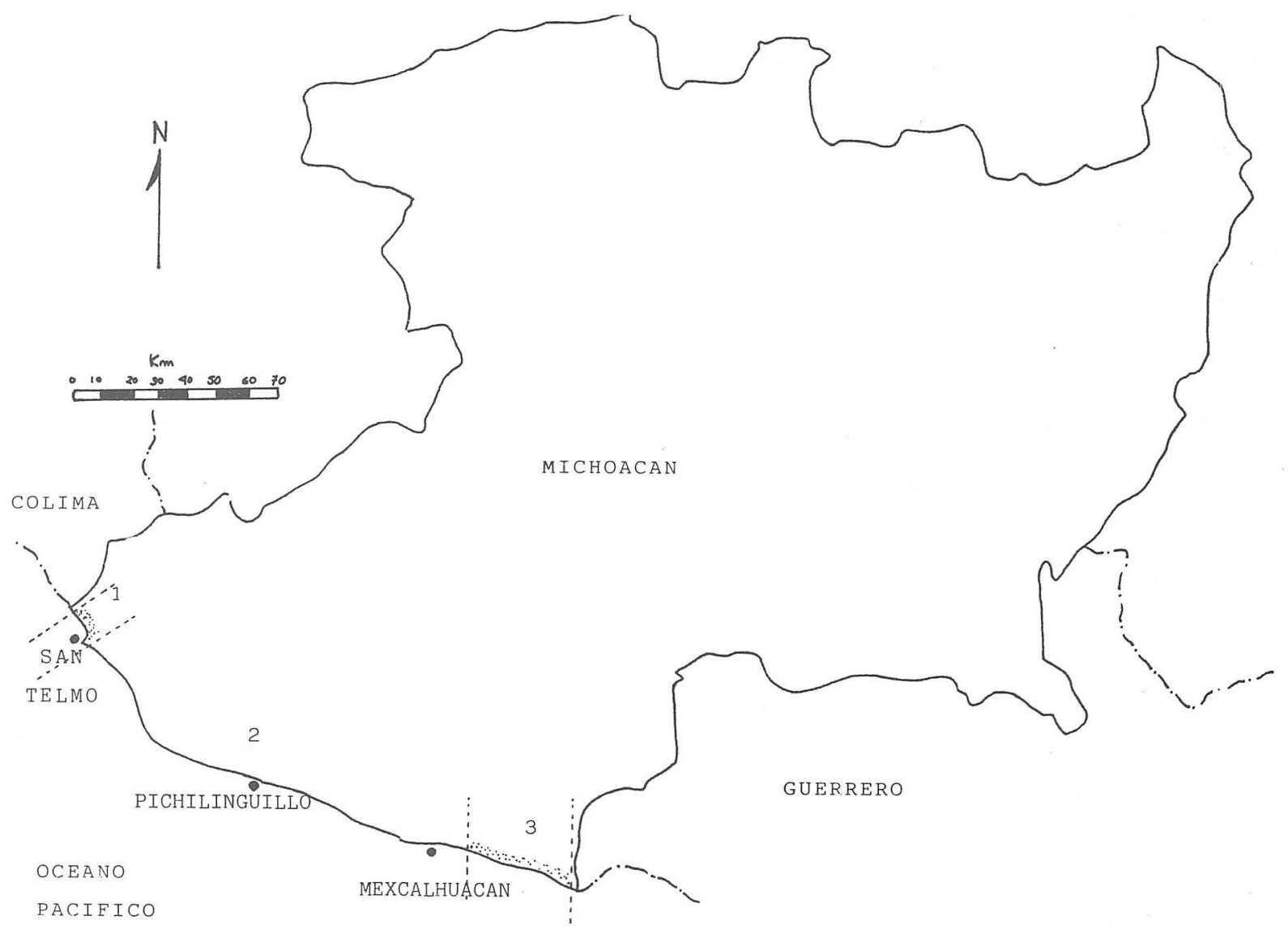

Fig. 1. Ubicación de localidades. 


\section{MÉTODO}

Las recolecciones se llevaron a cabo siguiendo las recomendaciones de Abbott y Dawson (1978), Taylor (1960) y Flores-Pedroche (1978); incluyen poblaciones de la zona mesomareal. Dichas muestras fueron conservadas en formol neutro al $4 \%$ y transportadas al laboratorio en donde el tratamiento taxonómico se realizó con: la separación genérica del material y el análisis de literatura, reunión de caracteres y elaboración de listados de atributos (Flores-Pedroche, 1981).

Los parámetros observados en cada uno de los ejemplares de las diferentes especies fueron colocados en orden decreciente de acuerdo a una importancia relativa dada por: a) naturaleza del carácter (morfológico, anatómico, reproductivo); b) constancia en los especímenes y c) facilidad de observación. Utilizando este orden se elaboraron las diagnosis correspondientes. Asimismo, las claves se realizaron empleando algunos caracteres diagnósticos de orden práctico, más no por ello irrelevantes.

Fueron preparadas laminillas con gelatina glicerinada y un poco de cristal violeta. Los dibujos se realizaron con ayuda de cámara clara y corresponden al primer autor del presente trabajo.

Los números de muestra citados pertenecen a ejemplares en líquido depositados en la colección ficológica de la Universidad Autónoma Metropolitana-Iztapalapa (UAMI) y las siglas que preceden al número consecutivo indican la procedencia (FM= Ficoflora de Michoacán).

\section{RESULTADOS}

$\begin{array}{ll}\text { Sistemática (Kylin, 1956) } \\ \text { División } & \text { RHODOPHYTA } \\ \text { Clase } & \text { FLORIDEOPHYCEAE } \\ \text { Orden } & \text { CERAMIALES } \\ \text { Familia } & \text { RHODOMELACEAE } \\ \text { Grupo } & \text { POLYSIPHONIA } \\ \text { Género } & \text { Polysiphonia Greville } \\ & \text { Polysiphonia confusa Hollenberg } \\ & \text { Polysiphonia scopulorum Harvey } \\ & \text { Polysiphonia sphaerocarpa Boergesen } \\ & \text { Polysiphonia sp. } \\ & \text { Tayloriella Kylin } \\ \text { Género } & \text { Tayloriella dictyurus (J.Ag.) Kylin } \\ & \text { HERPOSIPHONIA } \\ \text { Grupo } & \text { Herposiphonia Naegeli } \\ \text { Género } & \text { Herposiphonia littoralis Hollenberg } \\ & \text { Herposiphonia plumula(J.Ag.) Holleng. } \\ & \text { Herposiphonia tenella (C.Ag.) Ambronn }\end{array}$




$\begin{array}{ll}\text { Grupo } & \text { CHONDRIA } \\ \text { Género } & \text { Chondria C. Agardh } \\ & \text { Chondria arcuata Hollenberg } \\ & \text { Chondria dasyphylla (Woodward) C. Ag. } \\ & \text { Chondria decipiens Kylin } \\ \text { GruURo } & \text { Laurencia Lamouroux } \\ \text { Género } & \text { Laurencia clarionensis Setch. y Gard. } \\ & \text { Laurencia hancockii Dawson } \\ & \text { Laurencia voragina Taylor }\end{array}$

Clave genérica de la familia Rhodomelaceae en las costas de Michoacán

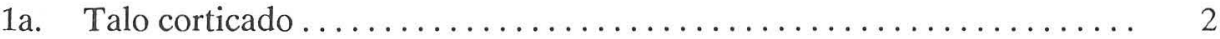

1b. Talo no corticado, si lo está, solamente en la base .............. 3 2a. Talo con 5 células pericentrales en corte transversal, y célula apical visible o sin depresión apical ..................................... 2b. Talo sin evidenciarse las células pericentrales en corte transversal, y la célula apical no es visible en la depresión apical............. Laurencia

3a. Talo con organización bilateral ................... Herposiphonia

3b. Talo con organización radial ......................... 4 4a. Presencia de tricoblastos, frecuentemente epifítica o epizoica . Polysiphonia 4b. Ausencia de tricoblastos, frecuentemente epilítica .......... Tayloriella

Polysiphonia Greville

Lista de caracteres diagnósticos

1. Diámetros del talo, tanto de ramas erectas como postradas.

2. Proporción de los segmentos en ramas erectas y rastreras.

3. Número de células pericentrales (en la parte media de los ejes).

4. Tipo de rizoides y de donde nacen.

5. Presencia y frecuencia de tricoblastos y/o cicatríces celulares.

6. Tipo de ramificación.

7. Hábito del talo.

8. Donde crecen (epífitas o saxícolas).

9. Dimensiones y disposición de los tetrasporangios.

10. Origen de las ramas (endo o exógenamente).

11. Color.

12. Dimensiones del cistocarpo.

13. Dimensiones del espermatangio.

14. Presencia o ausencia de células estériles en el espermatángio. 
15. Presencia o ausencia y grado de corticación.

Referencias: Hollenberg, 1961, 1968 y 1968a; Hollenberg y Norris, 1977; Womersley, 1979.

\section{Clave para las especies del género Polysiphonia}

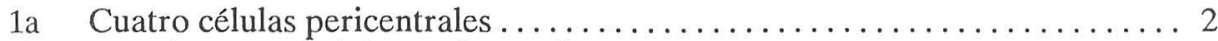

$1 b \quad$ Más de cuatro células pericentrales ....................... 3

2a. Ramas erectas con tricoblastos, cicatrices celulares o una rama en cada segmento, excepto cerca de la base ................... P. sphaerocarpa 2b. Ramas erectas con algunos segmentos sin tricoblastos, cicatrices celulares o

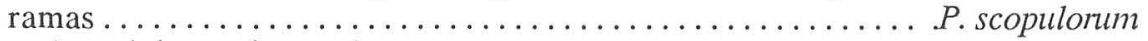

3a. Ocho células pericentrales......................... confusa 3b. Once células pericentrales .................... Polysiphonia sp.

Polysiphonia confusa Hollenberg. Lámina I

Alga con eje rastrero de diámetro entre $110-235 \mu \mathrm{m}$, segmentos 2.5 más anchos que largos. Ejes erectos con diámetro de 93- 112 $\mu$ m y sus segmentos 3.0 más anchos que largos. Presenta 8 células pericentrales; se fija al sustrato por rizoides unicelulares que nacen en conexión intercelular con las células pericentrales del eje rastrero. Los ejes erectos tienen en sus ápices tricoblastos, pero éstos no en una secuencia de uno por segmento. El talo tiene ramificación pseudodicotómica, cilíndrico, crece epifíticamente. Tetrasporangios con un diámetro de $37-58 \mu \mathrm{m}$ ordenándose en series espirales. Ramas erectas exógenas; la planta es de color rojo tenue. En el material revisado no se observaron ni cistocarpos ni espermatangios. Ninguna parte del talo presenta corticación.

Referencias: Abbott y Hollenberg, 1976 p.696 fig.643; Hollenberg, 1961 p.350 lám.1 fig.5; Sentíes, 1985 p.20 lám.1; Dreckmann, 1987 p.119 lám.XLII, figs. 1-4

Muestras: Pichilinguillo: FM 45 (plataforma rocosa). San Telmo: FM 17 (punta rocosa).

Nota: Esta alga puede ser confundida con P. hendryi Gardner pero los caracteres que las diferencia y que se toman en cuenta son: el número de células pericentrales (10-12) y el nacimiento de los rizoides es en conexión abierta, los cuales son determinantes para $P$. hendryi.

Polysiphonia scopulonum Harvey. Lámina II

Alga con eje rastrero de diámetro entre $62-125 \mu \mathrm{m}$, segmentos 1.5 más anchos que largos. Ejes erectos con diámetro de $25-50 \mu \mathrm{m}$ y sus segmentos 1.5 más anchos que largos. Presenta 4 células pericentrales; se fija al sustrato por rizoides que nacen en conexión abierta con las células pericentrales. Algunas de las ramas erectas tienen tricoblastos en sus ápices pero no en secuencia de uno por segmento. El talo tiene 
Senties, 85 .
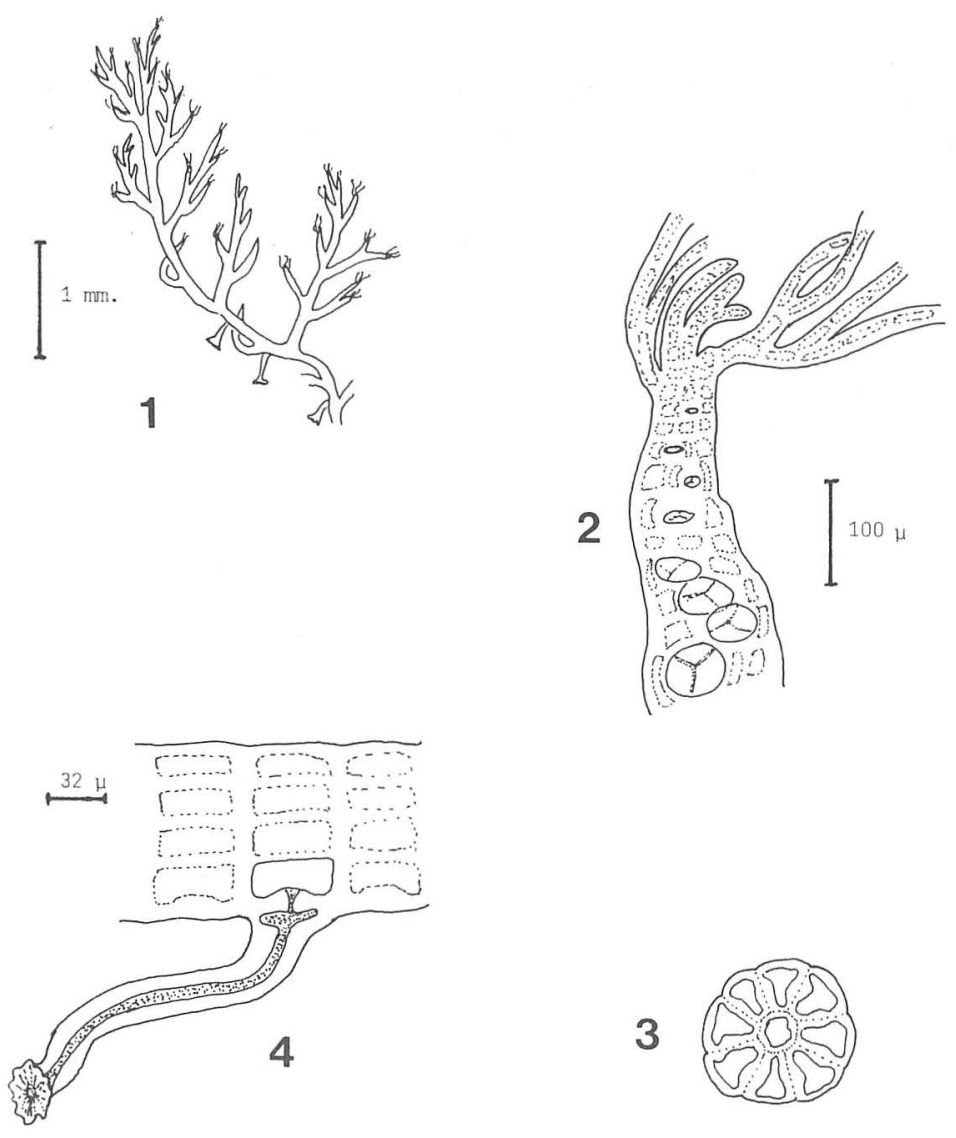

Lámina I

$$
50 \mu
$$

\section{camina}

Polysiphonia confusa. Fig.1.- Hábito. Fig.2.- Rama mostrundo la parte apical con tricoblastos $y$ el ondenamiento en espiral de los tetrasporangios. Fig. 3.- Corte transver sal observandose 8 células pericentrales y la célula axial. Fig.4.- Parte del talo con un rizoide naciendo en conexión intercelular de la célula pericentral. 

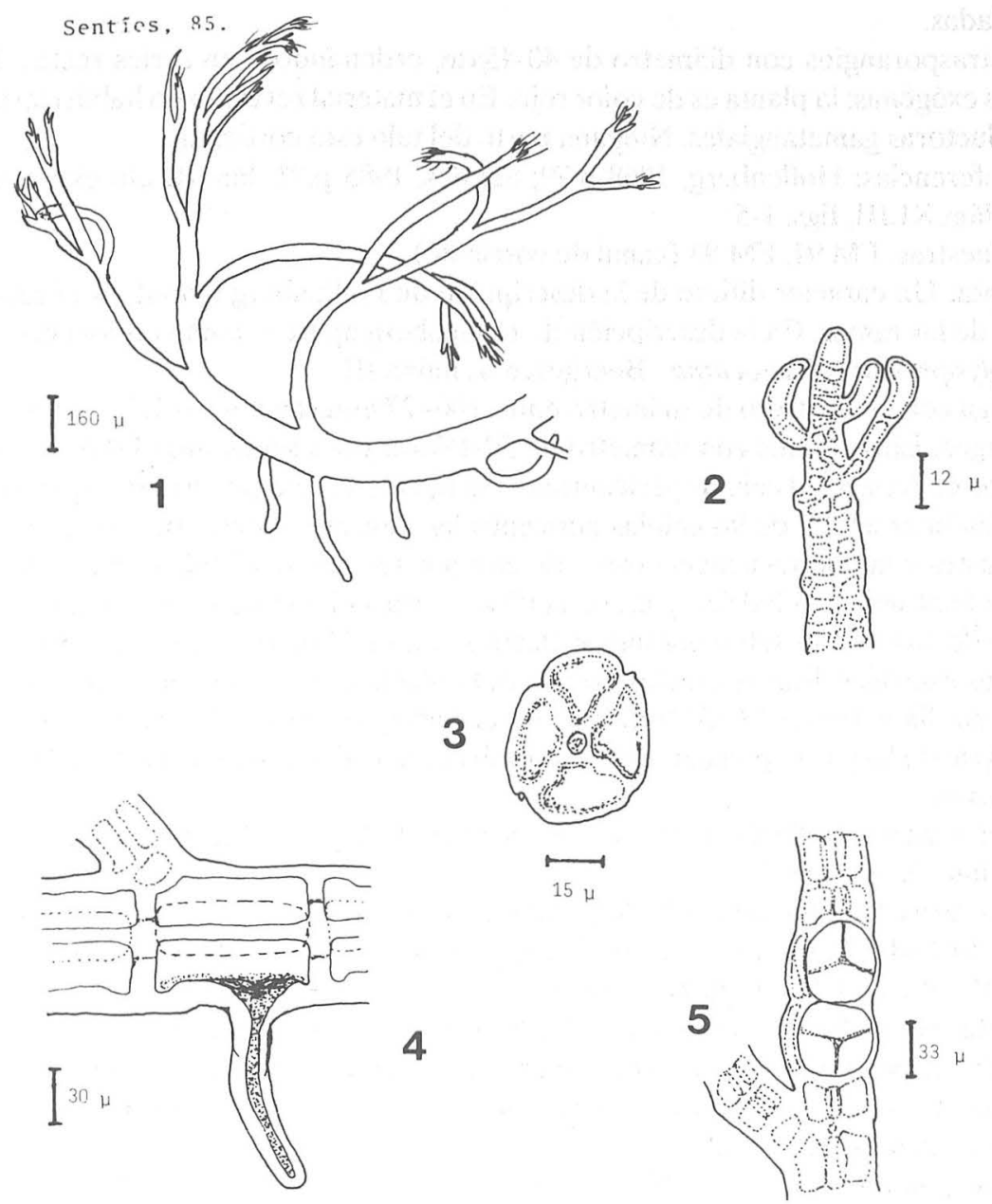

Lámina II

Polysinhonia scopulorur. Fig.1.- Hábito. Fig.2.- Parte apical con tricoblástos. Fig.3.Corte transversal observandose 4 cólulas pericentmles y la célula axial. Fig.4.- Rizoide naciendo en conexión abierta de la célula pericentral. Fig.5.- Rana con tetrasporangios en hilera recta. 
ramificación pseudodicotómica, cilíndrico y crece epifíticamente sobre algas coralinas articuladas.

Tetrasporangios con diámetro de 40-45 $\mu \mathrm{m}$, ordenándose en series rectas. Ramas erectas exógenas; la planta es de color rojo. En el material revisado no había estructuras reproductoras gametangiales. Ninguna parte del talo esta corticado.

Referencias: Hollenberg, 1968 p.79; Sentíes, 1985 p.21, lám.II; Dreckmann, 1987 p.120, lám.XLIII, figs. 1-5

Muestras: FM 91, FM 93 (canal de corriente)

Nota: Un caracter difiere de la descripción de Hollenberg (1968), es el relativo al origen de las ramas. En la descripción de Hollenberg aparece como endógeno.

Polysiphonia sphaerocarpa Boergesen. Lámina III

Alga con eje rastrero de diámetro entre 100- 279 $\mu$ m,segmentos 1.5-1.9 más anchos que largos. Ejes erectos con diámetro de 50-167 $\mu$ m y sus segmentos 1-1.5 más anchos que largos. Presenta 4 células pericentrales; se fija al sustrato por rizoides que nacen en conexión intercelular de las células pericentrales. Los ejes erectos tienen en sus ápices tricoblastos con una secuencia espiral de uno por segmento. El talo tiene ramificación pseudodicotómica, cilíndrico y crece epifítica o saxícolamente sobre sustrato de carbonato de calcio. Los tetrasporangios alcanzan hasta $70 \mu \mathrm{m}$ de diámetro, ordenándose en series espirales. Ramas erectas exógenas; la planta es de color rojo. Los cistocarpos tienen un diámetro de $279-372 \mu \mathrm{m}$. Los espermatangios miden de ancho $23-69 \mu \mathrm{m}$ por 46-184 $\mu \mathrm{m}$ de largo, no presentan células estériles apicales. Ninguna parte del talo tiene corticación.

Referencias: Hollenberg, 1968a p.87; Sentíes, 1985 p. 22, lám.III; Dreckmann, 1987 p. 120, lám. XLIV, figs. 1-5

Muestras: Pichilinguillo: FM 44 (punta rocosa), FM 46, FM 47 (plataforma rocosa), FM 60, FM 68, FM 69 (canal de corriente). San Telmo: FM 2, FM 3 (risco), FM 6, FM 9, FM 18, FM 19, FM 20 (punta rocosa).

Nota: Hollenberg menciona que $P$. sphaerocarpa se puede confundir con $P$. simplex Hollenberg, pero esta última generalmente esta representada por plantas grandes y básicamente postradas, con ramas asurgentes, mientras que $P$. sphaerocarpa es erecta con ramas postradas limitadas.

Polysiphonia sp. Lámina IV

Alga con eje rastrerode diámetro entre 80-105 $\mu \mathrm{m}$, segmentos 1-1.2 más anchos que largos. Los ejes erectos con un diámetro de $30-60 \mu \mathrm{m}$ y sus segmentos 1.5 más anchos que largos. Presenta 11 células pericentrales; se fija al sustrato por rizoides unicelulares que nacen en conexión intercelular de las células pericentrales. Los ejes erectos carecen de tricoblastos; el talo tiene una ramificación radial, cilíndrico, crece epifíticamente sobre algas coralinas articuladas. No presenta tetrasporangios. Ramas erectas exógenas; la planta es de color verde. En la muestra no se encontraron ni cistocarpos ni espermatangios. Ninguna parte del talo presenta corticación.

Referencias: Oliveira, 1969 p.130; Sentícs, 1985 p.23, lam IV 
Sentíes, 85 .
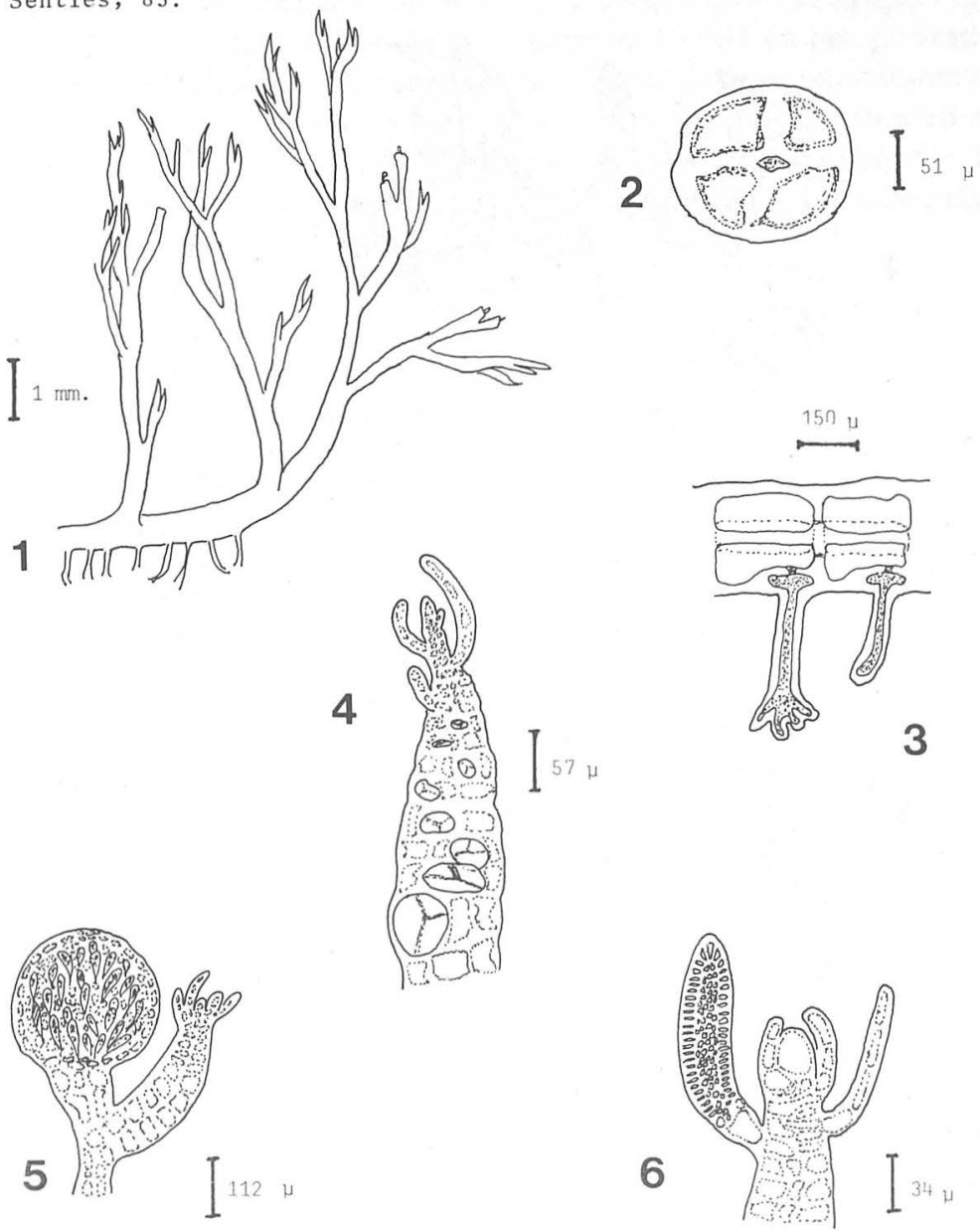

Lámina III

Polysiphonia sphaerocarpa. Fig.1.- Hábito. Fig.2.- Corte con 4 células Dericentrales. Fig.2.- Rizoides unicelulares naciendo en conexión intercelular de las céiulas pericen trales. Fig.4.- Rama tetrasponangial con los tetrasponangios en espiral. Fig.5.- Cisto carpo con las carposponas. Fir.6.- Parte apical mostrancio el espermatangio y tricolins. 
Senties, 85 .

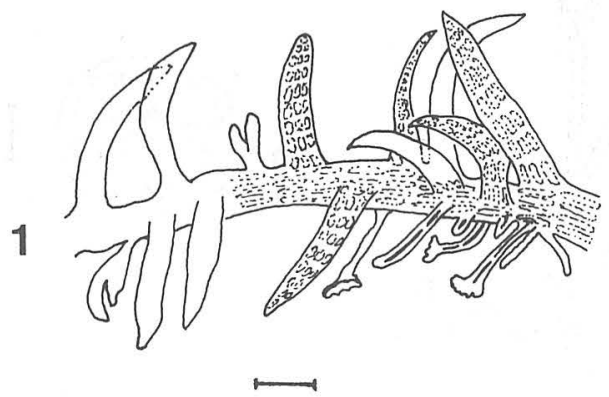

$88 \mu$
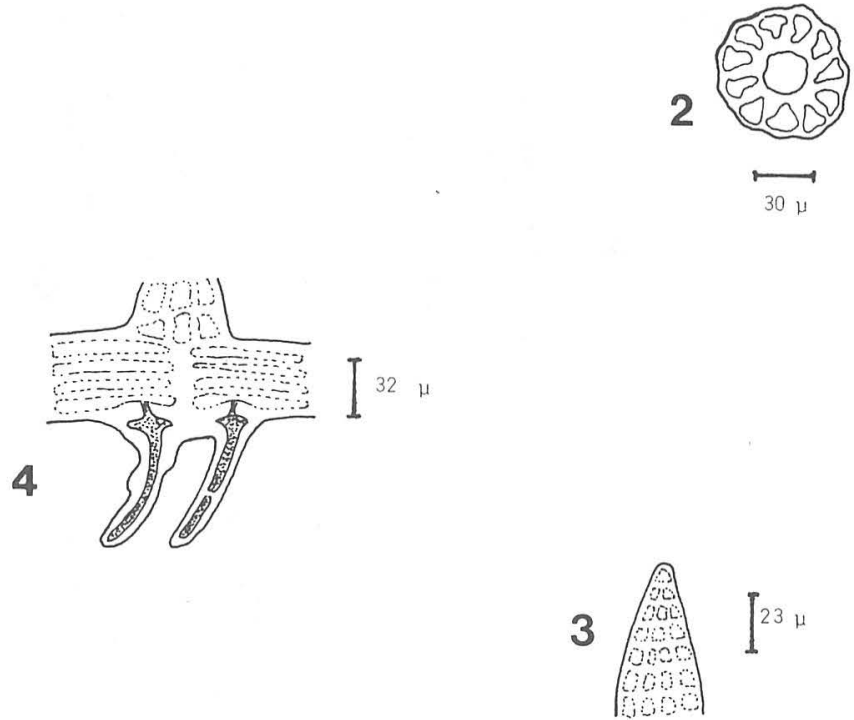

Lámina IV

Polysiphonia sp. Fig.1.- Hábito mostrando la disposición radial de las ramas erectas que parten de la porción postrada. Fig.2.- Corte transver al con 11 células pericentra les alrededor de la célula axial. Fig.3.- Parte apical. Fig.4.- Rizoides partiendo de las oélulas pericentrales en conexión intercelular. 


\section{Muestras: San Telmo FM 8 (punta rocosa)}

Nota: En esta alga todo parecería indicar que pertenece al grupo Herposiphonia, sin embargo por la ramificación radial se consideró dentro del grupo Polysiphonia. Ahora bien, dentro de este último cuyas especies presentan un eje postrado Oliveira reporta a $P$. howei Hollenbreg, la cual tiene apariencia de un representante del género Herposiphonia, pero hay caracteres que no concuerdan como: número de células pericentrales, diámetro de las ramas erectas y la presencia de tricoblastos. Por lo que esta alga puede ser una especie no descrita o bien requiere de un estudio más detallado en la región.

\section{Tayloriella Kylin}

\section{Lista de caracteres diagnósticos}

1. Diámetros, tanto del eje rastrero como de los erectos.

2. Número de células pericentrales (en parte media de los ejes).

3. Origen de las ramas (endo o exógenamente).

4. Hábito.

5. Altura del talo.

6. Diámetro de los tetrasporangios.

7. Posición de los cistocarpos.

8. Color.

Referencias: Dawson, 1963; Joly, 1967

Tayloriella dictyurus (J. Agardh) Kylin. Lámina V

Alga con un eje rastrero de 136- $237 \mu \mathrm{m}$ de diámetro, del cual parten ejes erectos con 364-437 $\mu \mathrm{m}$ de diámetro. Presenta đe 10 a 12 células pericentrales; ramas endógenas. La planta crece en matas sobre rocas, alcanza una altura aproximada de $2-5 \mathrm{~cm}$. Los tetrasporangios tienen un diámetro de 20-46 $\mu \mathrm{m}$, formando series de 6-11 en las ramas. Los cistocarpos están en parte lateral de las ramas terminales, siendo su diámetro de 233- 326 $\mu \mathrm{m}$. No se observaron espermatangios. Tiene un color café obscuro.

Referencias: Dawson, 1963 p.416 lám.4 fig. 5, lám 11 fig.4; Martinell, 1983 p.80; Sentíes, 1985 p. 25 lám. V; Dreckmann, 1987 p.122, lám. XLVI

Muestras: San Telmo: FM 2, FM47 (risco); FM 24, FM 26 (acantilado). Pichilinguillo: FM 41, FM 44 (punta rocosa); FM 69 (canal de coriente). Mexcalhuacan: FM 84, FM 89 (punta rocosa).

Nota: Dawson (1963) reporta que se desconocen las estructuras reproductoras gametangiales para el género, sin embargo, tanto en el trabajo de Martinell como en el presente se observaron cistocarpos.

Herposiphonia Naegeli

Lista de caracteres diagnósticos

1. Planos de ramificación.

2. Alternancia de las ramas determinadas e indeterminadas.

3. Longitud y diámetro de las ramas determinadas.

4. Diámetro del eje rastrero. 

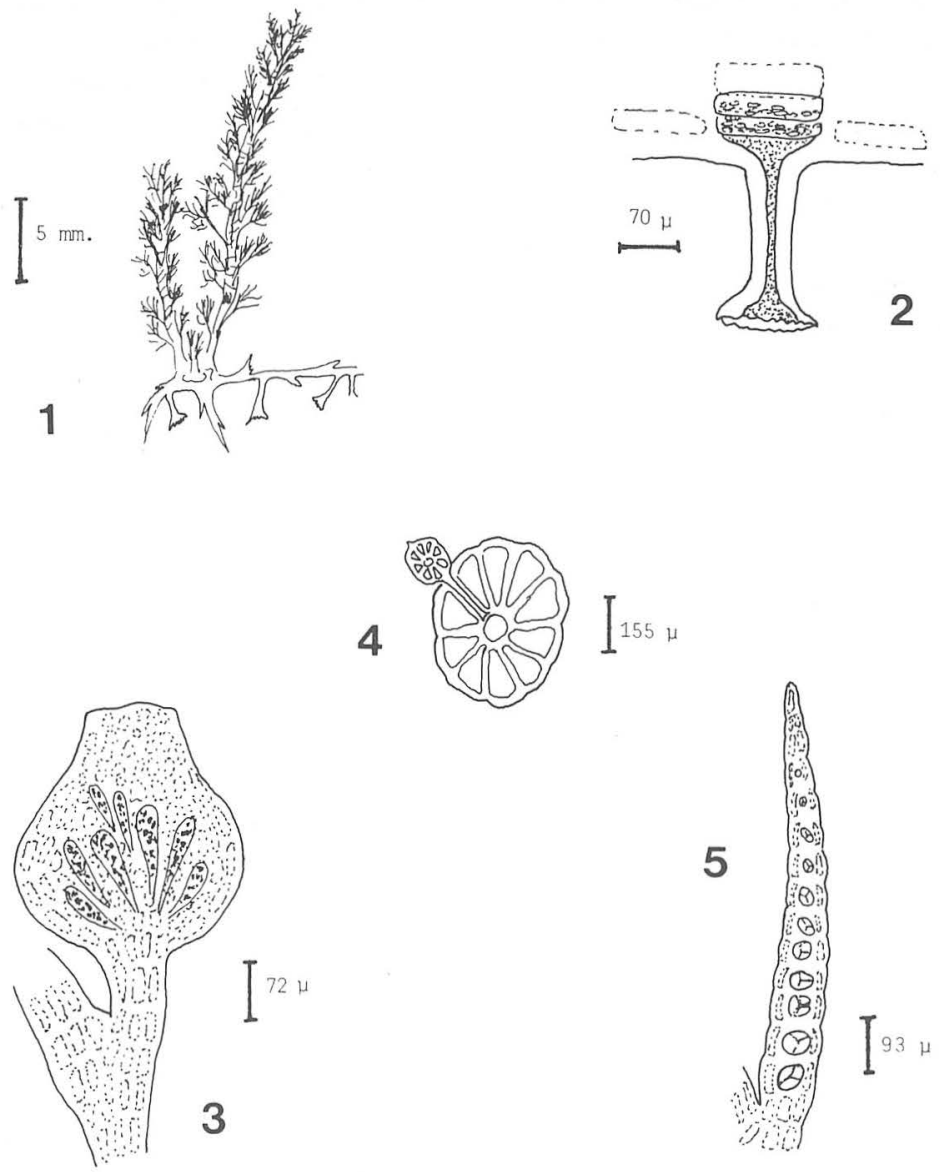

Lámina V

Tayloriella dictyurus. Fig.1.- Hábito. Fig.2.- Rizoide unicelular naciendo en conexión abierta de la célula pericentral. Fig.3.- Cistocarpo. Fig.4.- Corte transversal, mostran do 10 ó́lulas pericentrales y el nacimiento endógeno de una rama. Fig.5.- Disposición de los tetrasporangios. 
5. Relación (ancho-largo) de los segmentos.

6. Presencia o ausencia de tricoblastos.

7. Hábito.

8. Número de células pericentrales.

9. Diámetro de los tetrasporangios.

10. Dimensiones de los cistocarpos.

11. Dimensiones de los estiquídios espermatangiales.

12. Extensión del talo.

Referencias: Abbott y Hollenberg, 1976; Dawson, 1963.

\section{Clave para especies del género Herposiphonia}

1a. Ramas determinadas estríctamente dísticas en un plano........ H. plunnula

1b. Ramas determinadas dísticas pero en varios planos ................ 2a. Ramas con segmentos vacíos frecuentemente............. H. tenella 2b. Ramas con ramitas secundarias determinadas o indeterminadas en cada segmento .................................... littoralis

\section{Herposiphonia littoralis Hollenberg. Lámina VI}

Alga con eje rastrero del cual parten en su lado dorsal, en diferentes planos de ramificación, ramas determinadas e indeterminadas, teniendo una secuencia de tres determinadas por una indeterminada, no quedando ningún segmento vacío. Las ramas determinadas alcanzan una longitud de $1.3 \mathrm{~mm}$ y un diámetro de $75-100 \mu \mathrm{m}$; el eje rastrero comunmente de $112-225 \mu \mathrm{m}$ de diámetro; los segmentos son casi cuadrados, 1.5 más anchos que largos; las ramas determinadas tienen tricoblastos ramificados en el ápice. Es una planta saxícola, presenta de 10-13 células pericentrales. No se observaron estructuras reproductoras. El talo tiene $1 \mathrm{~cm}$ de extensión sobre el sustrato.

Referencias: Abbott y Hollenberg, 1976 p.718 fig. 666; Sentíes, 1985 p.28, lám. VI; Dreckmann, 1987 p. 124, lám. XLVIII, figs. 1-3.

Muestras: Pichilinguillo: FM 45 (plataforma rocosa).

Nota: Se menciona para esta especie, que las ramas determinadas tienen una longitud de $2-5 \mathrm{~mm}$ y un diámetro de $60-90 \mu \mathrm{m}$; el eje rastrero con $100-170 \mu \mathrm{m}$ de diámetro. Para este caso las medidas se amplian.

\section{Herposiphonia plumula (J. Agardh) Hollenberg. Lámina VII}

Alga con ramificación en un plano, Las ramas determinadas dísticas y en secuencia de tres leterminadas por una indeterminada, la cual se va alternando a cada lado del eje principal, las ramas determinadas van de $0.5-1.0 \mathrm{~mm}$ de longitud por $50-80 \mu \mathrm{m}$ en diámetro, siendo sus segmentos 1.5 más anchos que largos; carecen de tricoblastos. Es un alga epífita de coralinas, presenta 10 células pericentrales. Los tetrasporangios se dividen cruzadamente y miden $50-75 \mu \mathrm{m}$ de diámetro ya maduros. No se observaron estructuras reproductoras gametangiales. El talo tiene $8 \mathrm{~mm}$ de extensión. 
Sentíes, 85 .
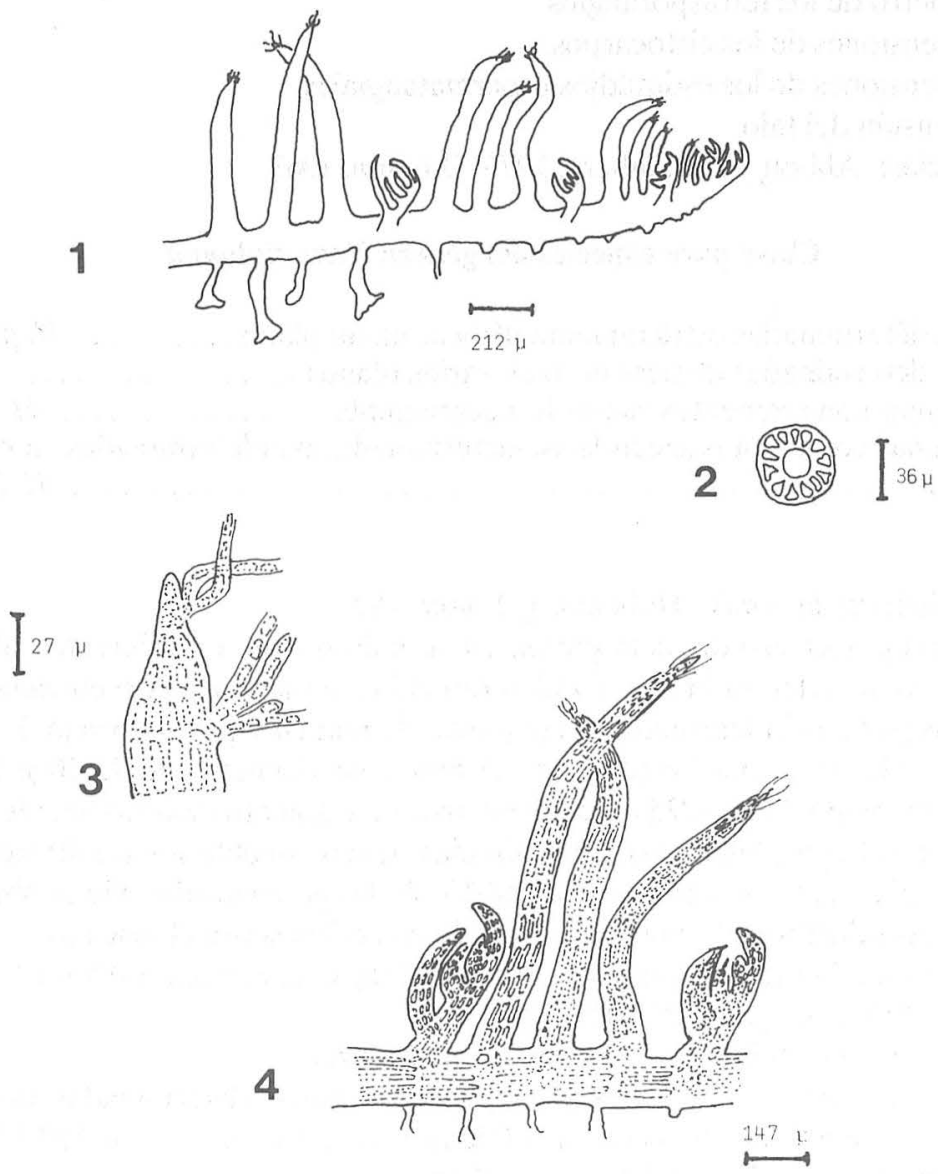

lámina VI

Herposiphonia littoralis. Fig.1. - Hábito mostrando la porción postrada y las ramas enéc tas.Fig.2.- Corte transversal con 13 células pericentrales. Fig.3. - Parte teminal de una rama determinada. Fig.4.- Parte del talo mostrando la secuencia de las namas determinadas con las indeterminadas. 
Sentíes, 85 .
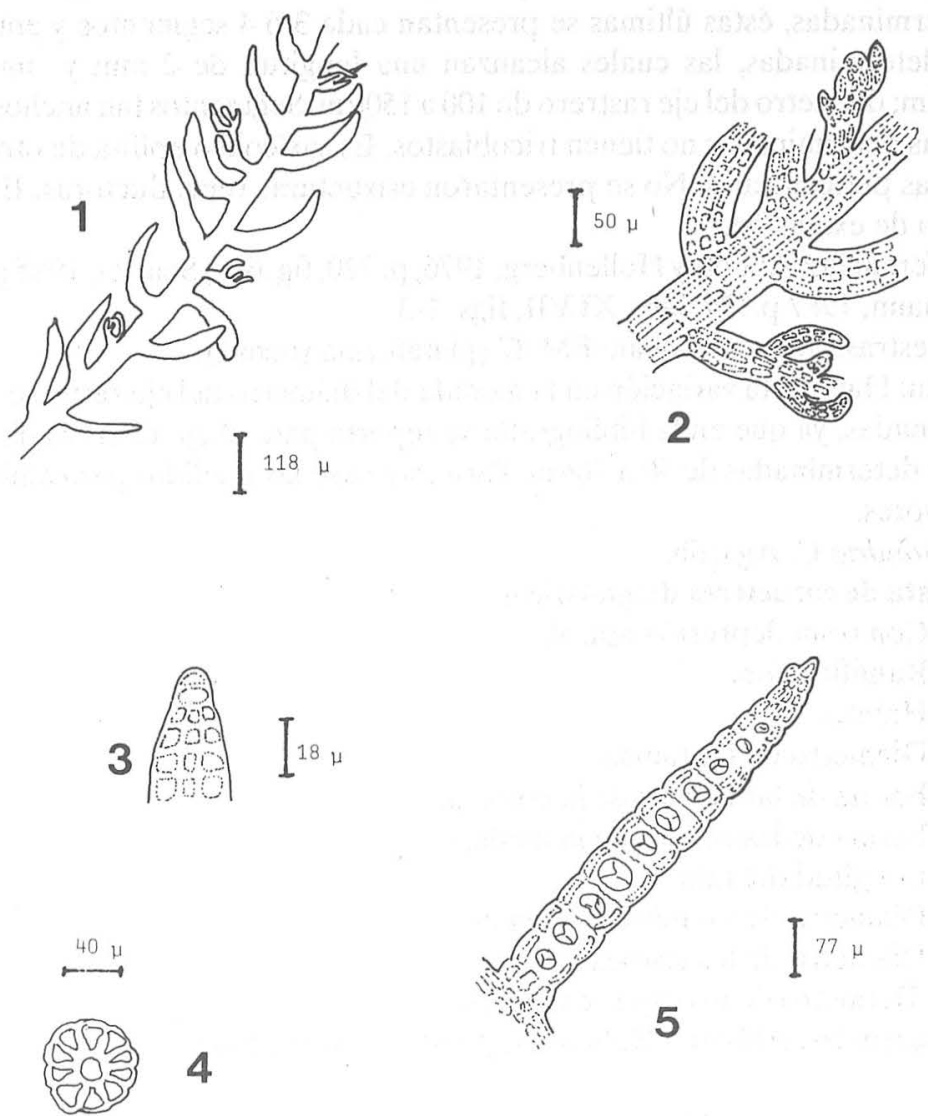

Lámina VII

Herposiphonia plumula. Fig.1.- Hábito con el eje central postrado y las namas enéctas Fig.2.- Detalle de la ramificación mostrando la secuencia de las ramas determinadas e indeterminadas. Fig.3.- Parte apical de una rama determinada. Fig.4.- Corte con 10 cé lu' as pericentrales. Fig.5.- Rama con tetrasponangios en hilena recta. 
Referencias. Abbott y Hollenberg, 1976, p. 718, fig. 667; Sentíes, 1985 p.29, lám. VII. Muestras: Mexcalhuacan: FM 86 (plataforma rocosa).

Herposiphonia tenella (C.Agardh) Ambronn. Lámina VIII

Alga con ramificación en varios planos, con una alternancia de ramas determinadas e indeterminadas, éstas últimas se presentan cada 3 ó 4 segmentos y entre éstas 1 ó 2 ramas determinadas, las cuales alcanzan una longitud de $2 \mathrm{~mm}$ y un diámetro de $50-125 \mu \mathrm{m}$; diámetro del eje rastrero de 100 a $150 \mu \mathrm{m}$. Segmentos tan anchos como largos; las ramas determinadas no tienen tricoblastos. Es saxícola o epífita de otras algas; tiene 10 células pericentrales. No se presentaron estructuras reproductoras. El talo tiene de 5-10 mm de extensión.

Referencias: Abbott y Hollenberg, 1976, p. 720, fig. 668; Sentíes, 1985 p.30 lám. VIII; Dreckmann, 1987 p.124, lám. XLVII, figs. 1-3.

Muestras.- Mexcalhuacan: FM 87 (plataforma rocosa).

Nota: Hay cierta variación en la medida del diámetro del eje rastrero y de las ramas determinadas, ya que en la bibliografía se reporta para el eje rastrero de 70 a $100 \mu \mathrm{m}$ y para las determinadas de 40 a $70 \mu \mathrm{m}$. Para este caso las medidas para ambos caracteres son mayores.

\section{Chondria C. Agardh}

Lista de caracteres diagnósticos

1. Con o sin depresión apical.

2. Ramificación.

3. Hábito.

4. Diámetro de las ramas.

5. Forma de las células de la corteza.

6. Forma de las células de la médula.

7. Longitud del talo.

8. Diámetro de los tetrasporangios.

9. Diámetro de los cistocarpos.

10. Diámetro de los espermatangios.

Referencias: Abbott y Hollenberg, 1976; Dawson, 1963.

\section{Clave para especies del género Chondria}

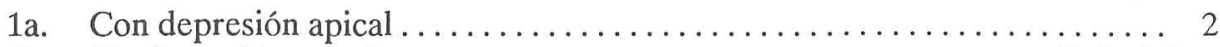

1b. Sin depresión apical ............................. decipiens

2a. Talo con ejes principales curvos .................. C. arcuata

2b. Talo con ejes principales rectos y rígidos ............. C. dasyphylla

\section{Chondria arcuata Hollenberg}

Alga con depresión apical en sus ramas, ramificación irregular, alterna o radial. Talo cilíndrico con ejes principales notoriamente curvos al igual que las escasas ramitas laterales. Diámetro de las ramas de 0.4 a $0.5 \mathrm{~mm}$. En corte transversal las células 


\section{RHODOMELACEAE EN MICHOACAN}

Senties, 85 .
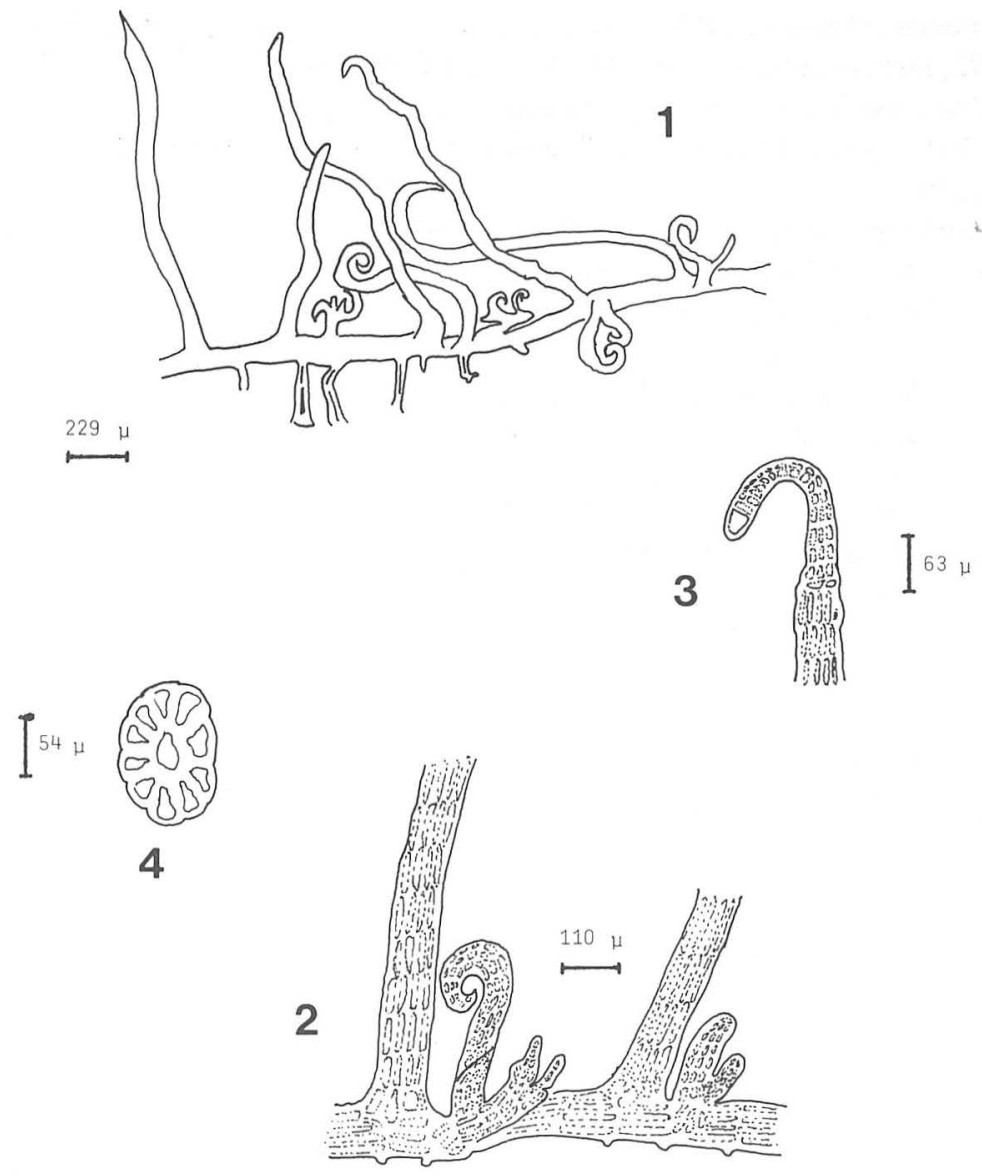

Lámina VIII

Herposiphonia tenella Fig.1. - Hábito. Fig.2.- Detalle del talo con la

altemación de una rama deteminada con una indeterminada. Fig.3.- Parte teminal de una rama determinada. Fig.4.- Corte transversal con 10 células pericentrales. 
corticales tienen forma cuadrada, algunas acuñadas y las células medulares son isodiamétricas. La altura del talo varía de 2.0-2.5 (3.0) $\mathrm{cm}$. Se observaron discos espermatangiales (150-170 $\mu \mathrm{m}$ de diámetro) por debajo de las depresiones apicales y en la base de los tricoblastos, localizados en ramas cortas y más globosas que el resto. No se presentaron otro tipo de estructuras reproductoras.

Referencias: Dawson, 1963, p. 442, lám. 42: figs. 1-3; Abbott y Hollenberg, 1976, p. 724, Fig.672; Dreckmann, 1987 p. 126, lám. XLIX, figs. 1-4.

Muestras: San Telmo: FM 7 (punta rocosa).

Nota: Esta especie fué considerada por Dreckmann (1987) como un nuevo registro para el estado.

Chondria dasyphylla (Woodward) C. Agardh. Lámina IX

Alga con depresión apical en sus ramas, ramificación alterna radial. Talo cilíndrico, ejes más o menos rígidos, con diámetro de 0.4-0.8 mm. En corte transversal las células corticales tienen forma cuadrada y las células medulares son isodiamétricas. La altura del talo varía de $1.5-2.5 \mathrm{~cm}$; los tetrasporangios tienen un diámetro de 60 a $95 \mu \mathrm{m}$. No se presentaron estructuras reproductoras gametangiales.

Referencias: Abbott y Hollenberg, 1976 p. 726, fig. 674; Dawson, 1963, p. 444, lám. 15, fig. 4, lám. 38; Sentíes, 1985 p. 33, lam.IX.

Muestras: Mexcalhuacan: FM 87 (plataforma rocosa); FM 93, FM 95 (canal de corriente); FM 99 (risco).

Nota: En la bibliografía se menciona que el diámetro de los tetrasporangios es de $140-165 \mu \mathrm{m}$ y en este caso alcanzan $60-95 \mu \mathrm{m}$.

Chondria decipiens Kylin. Lámina X

Alga sin depresión apical en sus ramas, ramificación multivariada irregularmente. Talo arbustivo, ejes flácidos, con diámetro de 0.5-0.8 mm. En corte transversal la mayoría de las células corticales son cuadradas, con algunas acuñadas y en la médula, isodiamétricas. Longitud del talo de $2 \mathrm{~cm}$. En el material revisado no hubo estructuras reproductoras.

Referencias: Abbott y Hollenberg, 1976, p. 726, fig. 675; Dawson, 1963, p. 446, lám. 7, fig. 1; Sentíes, 1985 p. 34, lám. X.

Muestras: Mexcalhuacan: FM 84 (plataforma rocosa).

Nota: La talla reportada es de $8-16 \mathrm{~cm}$, sin embargo en este caso sólo llega a $2 \mathrm{~cm}$.

Laurencia Lamouroux

Lista de caracteres diagnósticos

1. Ramificación.

2. Longitud del talo.

3. Diámetro de la parte basal y erecta.

4. Hábito.

5. Forma y diámetro de las células de la corteza.

6. Presencia o ausencia de uniones intercelulares secundarias en las células corticales. 


\section{RHODOMELACEAE EN MICHOACAN}

Sentíes, 85 .
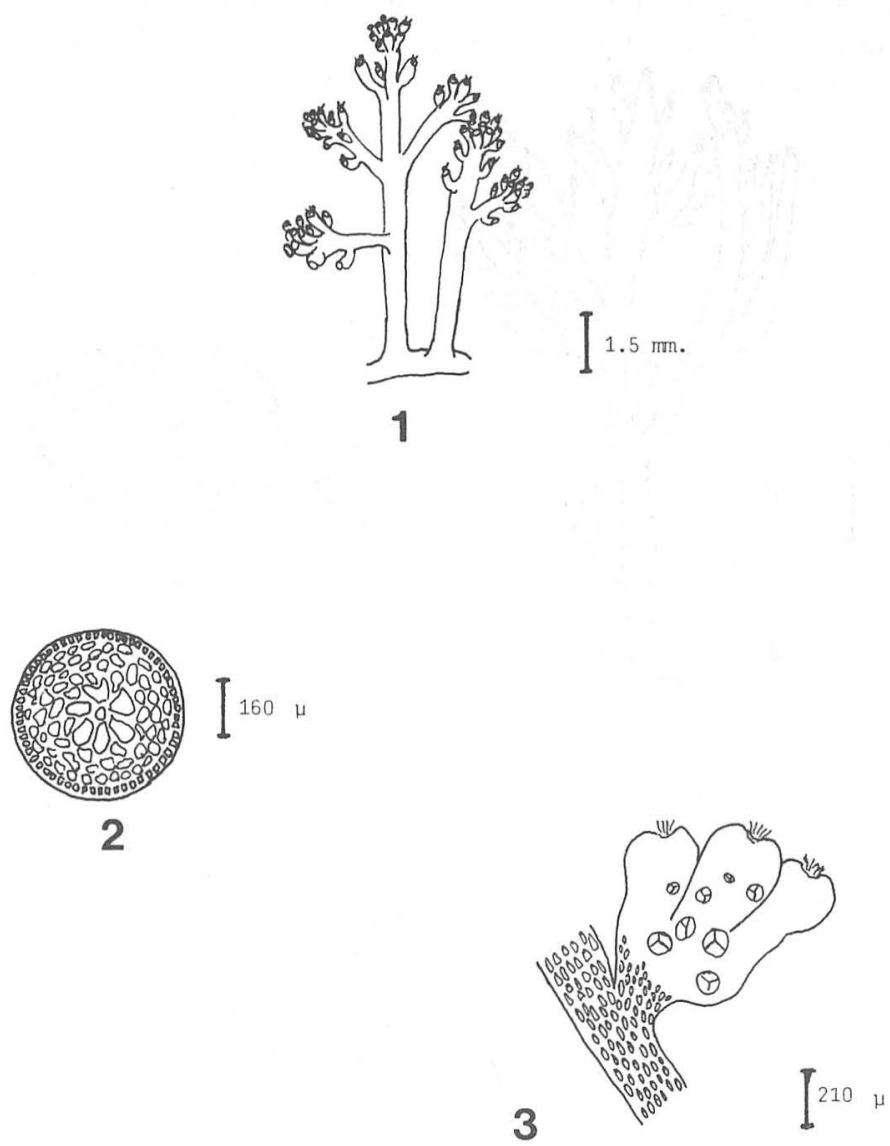

Lämina IX

Chondria dasyphylla. Fig.1.- Detalle mostrando tipo de ranificación y ramas teminales. Fig.2.- Corte transversal del talo. Fig.3.- Rama con tetrasporangios. 
Sentîes, 85 .
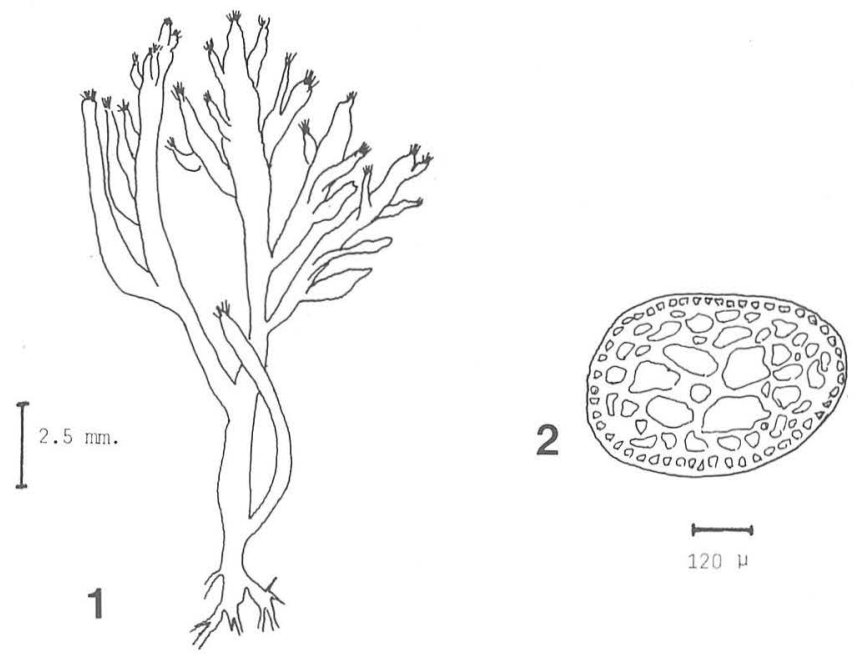

$120 \mu$

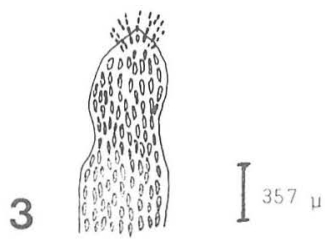

Lámina $X$

Chondria decipiens. Fir.1. - Hábi to mostrando el tipo de ramificación. Fig.2.- Conte transversal con las 5 células pericentrales grandes. Fig.3.- Parte teminal de una rama on pequeños tricoblastos. 
7. Forma y diámetro de las células de la médula.

8. Presencia o ausencia de engrosamientos lenticulares.

9. Disposición y diámetro de los tètrasporangios.

10. Forma y diámetro de los cistocarpos.

11. Forma y diámetro de los espermatangios.

Referencias: Abbott y Hollenberg, 1976; Saito y Womersley, 1974; Taylor, 1945.

Nota: Despues de haber terminado el trabajo, la Dra.M.T. Fujii (com. pers.) sugiere además de los anteriores, dos caracteres adicionales: (cabe mencionar, que por razones diversas no fue posible constatar éstos en el material estudiado).

1. Inserción de los tetrasporangios en la célula pericentral (adaxial o abaxialmente).

2. Forma del receptáculo espermatangial (taza o bolsa).

\section{Clave para las especies del género Laurencia}

1a. Plantas con menos de $350 \mu \mathrm{m}$ de diámetro, talo principalmente rastrero ........

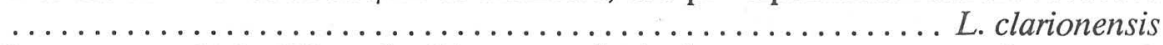

1b. Plantas con más de $350 \mu \mathrm{m}$ de diámetro, principalmente erecta o ascendente. . 2 $2 \mathrm{a}$. Ejes erectos con un diámetro cercano a $720 \mu \mathrm{m}$, con ramificación frecuentemente unilateral, pero algunas ocasiones irregular multivariada .. . L. hancockii 2b. Ejes erectos con un diámetro cercano a $1000 \mu \mathrm{m}$, con ramificación regular

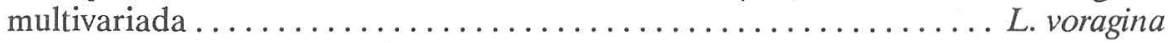

\section{Laurencia clarionensis Setchell y Gardner. Lámina XI}

Alga con ramificación irregular, talo de $2-5 \mathrm{~mm}$ de altura, éste se compone de una parte rastrera con diámetro de $400-700 \mu \mathrm{m}$ y una parte de ramas erectas que generalmente no están ramificadas, con un diámetro de $200-500 \mu \mathrm{m}$. Todo el talo es cilíndrico y forma pequeñas matas junto con otras algas. Observando su médula parenquimatosa, las células corticales son redondas o isodiamétricas con 25-30 $\mu \mathrm{m}$ de diámetro, presentan uniones intercelulares secundarias. Engrosamientos lenticulares presentes. Los tetrasporangios se disponen en la parte apical de las ramas erectas de forma paralela al eje central y miden de 33-75 $\mu \mathrm{m}$ de diámetro. En el material no se observaron estructuras reproductoras gametangiales.

Referencias: Dawson, 1963, p. 450, lám. 45, figs. 3-5; Sentíes, 1985 p. 37, lám. XI.

Muestras: Mexcalhuacan: FM 87 (plataforma rocosa); FM 91 (canal de corriente).

Laurencia hancockii Dawson. Lámina XII

Alga con ramificación unilateral y en ocasiones irregular multivariada, altura máxima de $1 \mathrm{~cm}$. El talo se compone de ejes postrados con diámetro de 270-600 $\mu \mathrm{m}$ y de ejes erectos con 380-720 $\mu \mathrm{m}$ de diámetro; crece en pequeñas matas sobre rocas. En corte transversal se aprecian las células corticales cuadradas o acuñadas de $12-28 \mu \mathrm{m}$ de diámetro, presentando uniones intercelulares secundarias. Las células medulares 
Senties, 85 .
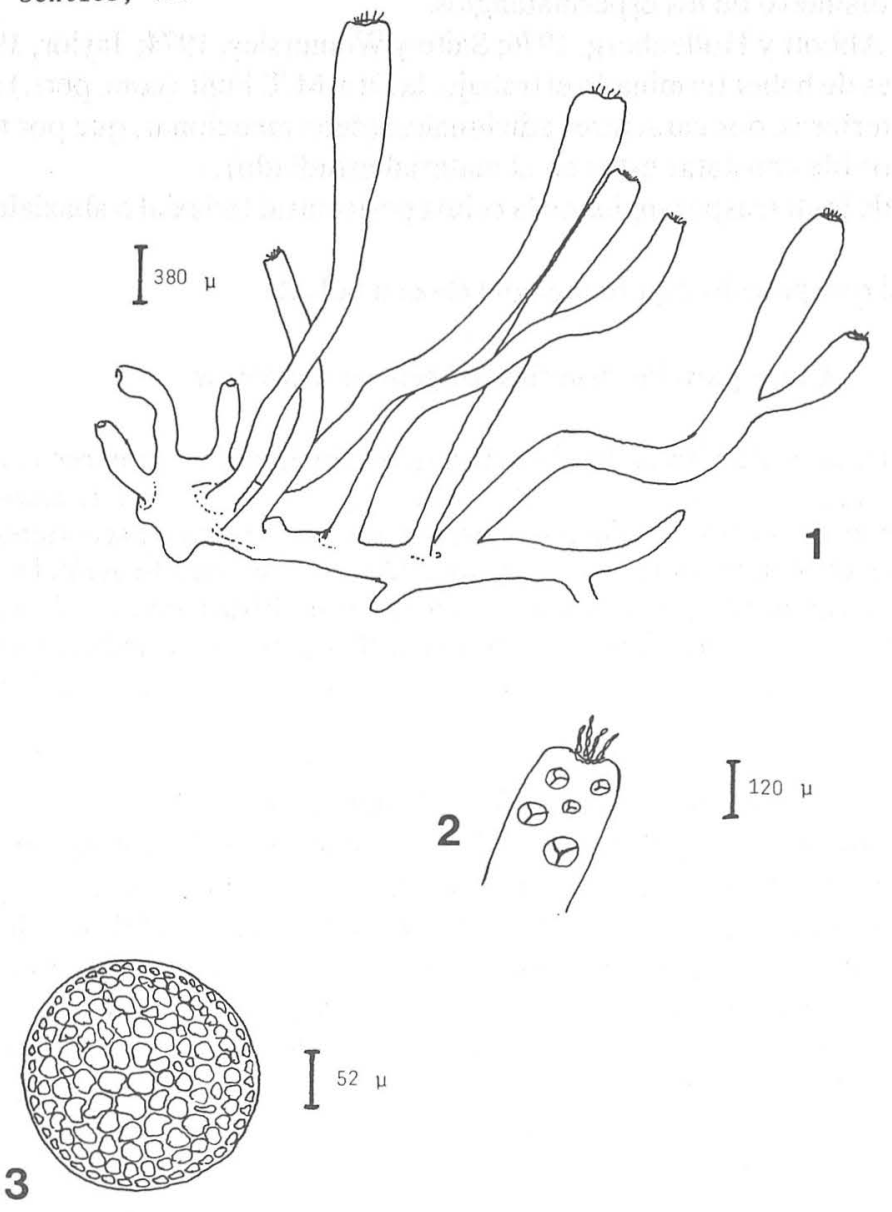

Lámina XI

Launencia clarionensis. Fig.1.- Hábito mostrando un eje rastrero y ramas erectas. Fig.2.- Parte apical de una rama con tetrasporangios. Fig.3.- Corte transversal del talo. 
redondas e isodiamétricas con $32-76 \mu \mathrm{m}$ de diámetro. Engrosamientos lenticulares ausentes. Presenta tetrasporangios en los ápices de las ramas terminales, dispuestos en forma paralela al eje central, su diámetro oscila entre $30-95 \mu \mathrm{m}$. Se observaron cistocarpos en corte transversal de forma ovoidal con $306 \mu \mathrm{m}$ de diámetro, conteniendo muchas carposporas. Los espermatangios no fueron observados.

Referencias: Dawson, 1963, p. 452, lám. 24, fig. 1; Sentíes, 1985 p. 38, lám. XII; Dreckmann, 1987 p. 127, lám. L, figs. 1- 3.

Muestras: San Telmo: FM 7, FM 16, FM 17, FM 20, FM 21, (punta rocosa). Pichilinguillo: FM 45, FM 46 (plataforma rocosa); FM 52, FM 61, FM 68, FM 69 (canal de corriente). Mexcalhuacan: FM 86, FM 89 (plataforma rocosa).

Nota: En la bibliografía se dice que las ramas erectas tienen un diámetro de $300-500 \mu \mathrm{m}$ y en estos ejemplares se alcanza hasta $720 \mu \mathrm{m}$. Además Dawson (1963) reporta que no se han observado plantas sexuales y para este caso se encontraron cistocarpos maduros.

Laurencia voragina Taylor. Lámina XIII

Planta con ramificación radial y alterna en algunos ejes, alcanza una altura de $2 \mathrm{~cm}$. El talo tiene una parte basal con un diámetro de 750-900 $\mu \mathrm{m}$, y una parte erecta de $500-1000 \mu \mathrm{m}$ de diámetro. Todo el talo es cilíndrico, crece formando matas o arbustos sobre las rocas. Las células de la corteza tienen una forma cuadrada o acuñada con un diámetro de 10-27 $\mu \mathrm{m}$, presentan conecciones intercelulares secundarias. Las células de la médula son isodiamétricas con 30-70 $\mu \mathrm{m}$ de diámetro. Engrosamientos lenticulares ausentes. En el material revisado no se observaron estructuras reproductoras.

Referencias: Dawson, 1963, p. 470, lám. 29, fig. 3; Taylor, 1945, p. 290, pl. 46, fig. 2; Sentíes, 1985 p. 39, lám. XIII; Dreckmann, 1987 p. 128, lám. LI, figs. 1 y 2.

Muestras: San Telmo: FM 18, FM 19, FM 20 (punta rocosa);Pichilinguillo: FM 41 (punta rocosa); Mexcalhuacan: FM 92, FM 94, FM 95 (canal de corriente).

\section{DISCUSIÓN}

Aspectos taxonómicos: durante la realización de las listas de los caracteres para cada uno de los géneros, los autores consultados seleccionan y ponderan los parámetros de formas diferentes y por lo tanto, éstos varían de un autor a otro. Sin embargo, la posibilidad de reunir aquí el mayor número de caracteres, proporcionó una mejor guía para la determinación y caracterización de las especies.

El haber evaluado, interpretado y ponderado las características presentes en algunos de los integrantes de la familia, permitió evidenciar la utilidad taxonómica que cada uno de ellos posee. Es decir, al quedar representada una secuencia de ponderación en las descripciones, se observó: a) el comportamiento del caracter a lo largo de las especies investigadas; b) que caracteres son de más peso para poder ubicar a cada uno 
Senties, 85 .
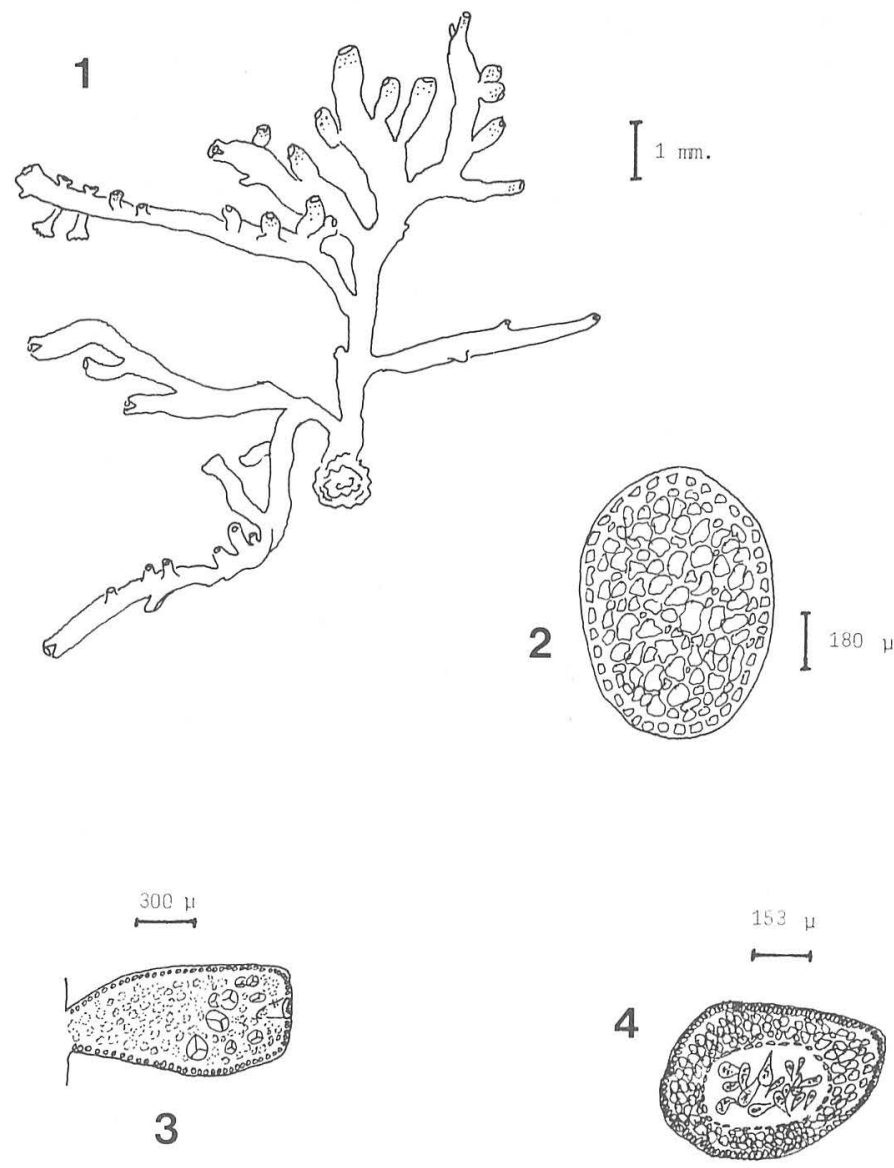

Lámina XII

Laurencia hancockii. Iig.1.- Hábito mostrando el tipo de ramificación. Fig.2.- Corte transversal. Fig.3.- Rana con tetrasponangios. Fig.4.- Conte transversal mostran do las carposponas. 
Sentíes, 85 .
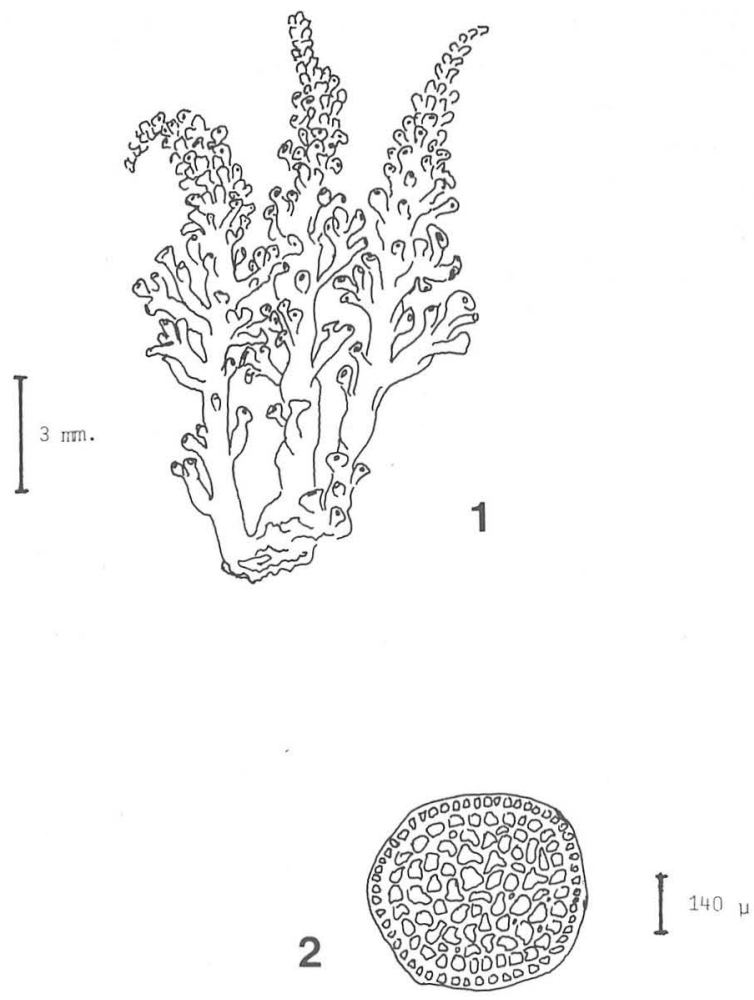

Támina XIII

Laurencia voragina. Fig.1.- Hábito, mostrando el tipo de ramificación. Fig.2.- Conte tnansversal del talo. 
de los especímenes en especies ya establecidas, y c) cual o cuales aportan información complementaria.

Así podemos observar, que para las especies representantes de los géneros Polysiphonia y Tayloriella el caracter que tiene más peso es el de las dimensiones y proporciones, tanto de ramas erectas como postradas, siendo el color el caracter que aporta un dato complementario. Para el género Herposiphonia, los atributos de más peso fueron: los planos de ramificación y la alternancia de las ramas determinadas e indeterminadas y como caracter de menor peso, las dimensiones de las estructuras reproductoras. En el caso del género Chondria, el atributo a ponderar fué la presencia o no de una depresión apical, siendo un caracter secundario, las dimensiones de las estructuras reproductoras. Para Laurencia, el tipo de ramificación representa la característica fundamental.

Las aseveraciones anteriores tienen su fundamento en el hecho de que todos los caracteres toman valor taxonómico al presentar tres condiciones: constancia, curva de variación estrecha y baja sensibilidad a los cambios ambientales (De la Sota, 1967).

Es cierto que la agrupación de los caracteres fue de mucha ayuda para la determinación y caracterización de las especies; al observar nuestros resultados, en el rubro de descripciones, nos encontramos con un caso en el cual no se llegó a determinar la especie (Polysiphonia sp.) dejándose indicados, lo más completo posible, los parámetros que ella presenta. Es importante resaltar que los caracteres que no concuerdar con la descripción de $P$. howeii, reportada por Oliveira (1969), y que es la especie con la que más semejanza tiene, son parámetros fundamentales que poseen un peso determinante dentro del género, como son: número de células pericentrales, diámetro de las ramas erectas y presencia de tricoblastos. Por lo que se hace necesario el análisis de otros especímenes más completos, en cuanto a los parámetros evaluados, o ampliar el tiempo de estudio para tener la oportunidad de observar un número mayor de ejemplares y así llevar a cabo una determinación acertada.

Aspectos ecológicos y de distribución: de todos los taxa de la familia reportados para el Pacífico tropical mexicano (Pedroche, com. pers.), sólo un 13.5\% han sido citados en el presente trabajo. Cabe destacar que este estudio aporta 6 nuevos registros para México en sus costas tropicales; Polysiphonia confusa, P. scopulonum, Chondria arcuata, C. dasyphylla, Laurencia clarionensis y L. hancockii. Estas mismas especies, tampoco habían sido dadas a conocer para otras zonas tropicales, lo cual implica que se amplió, tanto su distribución como el intervalo de variación para algunos de los caracteres. Estas seis especies representan un $42 \%$ del total de especies que aquí se consideran; este porcentaje es importante para el escaso estudio que existe de la ficoflora de México y de todo el Pacífico tropical.

Por otra parte, analizando los registros de la presencia- ausencia en cada una de las localidades del estado (tabla 1) observamos que hay tres posibilidades, en las que se agrupan las especies: 1) las que están en las 3 localidades; 2) las que están en 2 localidades y 3) aquellas que están sólo en una. 
Esta agrupación se interpreta de la siguiente manera: para el primer caso, existe a lo largo de la costa del Oceáno Pacífico tropical mexicano una afinidad florística, debido a las condiciones topográficas, ecológicas, físicas, etc., las cuales son muy similares para esa zona (González-González, 1980). Es por esta razón que encontramos especies comunes las cuales pueden manifestarse en muchos lugares y bajo diversas condiciones. Las especies que aparentemente están en tal generalidad son: Tayloriella dictyurus, Laurencia hancockii y $L$. voragina, y en las que aparentemente no se discriminan habitats.

La segunda posibilidad se debe, quizá a una diferencia en el sustrato rocoso que se encuentra en las localidades, el cual es similar para San Telmo y Pichilinguillo, existiendo un contraste con el presente en Mexcalhuacan. Aunque el tipo de roca no ha sido estudiado como factor determinante, sí puede considerarse fundamental para el establecimiento de las algas, sin tomar en cuenta sus propiedades físicas o químicas (Silva, 1979). Como ejemplos de esta situación podemos anotar a Polysiphonia confusa, $P$. sphaerocarpa y Herposiphonia littoralis. Otra explicación para esta presencia dual puede encontarse en aquellas especies que responden a presiones particulares del medio y se manifiestan poblacionalmente en ciertos ambientes, los que han sido trabajados parcialmente por Pedroche y González-González (1980), que proponen nueve ambientes generáles para las costas del Pacífico tropical mexicano, estas unidades son de utilidad para poder comparar y predecir la presencia de determinada especie. En este caso encontramos a: Polysiphonia scopulorum y Herposiphonia tenella.

Para el último grupo de especies las condiciones muy específicas ("microambientes"), principalmente en lo que a plataforma rocosa se refiere, que podrían evidenciarse en grietas, canales de corriente, oquedades, pozas de marea, entre otros, propician que estos establecimientos sean en muchas ocasiones particulares a ciertas localidades. Dentro de éstas tenemos para San Telmo; Polysiphonia sp. y Chondria arcuata y para Mexcalhuacan; Herposiphonia plumula, Chondria dasyphylla, C. decipiens y Laurencia clarionensis.

AgRADECIMIENTOS. Agradecemos a las Dras. Rosario de A. Braga y Mutue T. Fujii del Instituto de Botánica, Sâo Paulo, Brasil y al Dr. Hermilo Quero R. del Jardín Botánico, UNAM por sus valiosas sugerencias y la revisión crítica del manuscrito.

\section{LITERATURA CITADA}

ABBOTT, I.A. y E.Y. DAWSON. 1978. How to know the seaweeds. The pictured key nature series. Wm.

C. Brown Co. Publishers. 141p.

ABBOTT, I.A. y G.J. HOLLENBERG. 1976. Marine algae of California. Stanford University Press. 827p. ARDRE, F. 1967. Remarques sur la structure des Pterosiphonia (Rhodomélacées, Ceramiales) et leurs rapports systématiques avec les Polysiphonia. Revue Algologique 1: 37-77.

CORREA, M.Z. 1986. La división Chlorophyta en las costas del estado de Michoacán, Méx. Tesis, Fac. de Ciencias, UNAM. México. 80p. 
DAWSON, E.Y. 1963. Marine red algae of Pacific Mexico. Part. 8. Ceramiales: Dasyaceae, Rhodomelaceae. Nova Hedwigia 6: 401- 481, pls. 126(1)-171(46).

DE LA SOTA, E.1967. La taxonomía y la revolución en las ciencias biológicas. O.E.A. Serie de Biología, Monografía No.3, 86p.

DRECKMANN, E.K.M.1987. Algas marinas bénticas de playa San Telmo, Michoacán, México. Tesis, Fac. de Ciencias, UNAM. México. 170p.

FLORES-PEDROCHE,F. 1978. Estudio florístico preliminar de las macroalgas mesolitorales de las costas de la región de Chamela, Jalisco. Tesis, Fac. de Ciencias, UNAM. México, D.F. 110p.

FLORES-PEDROCHE, F. 1981. Los géneros Codium y Halimeda (Chlorophyta) en El Salvador, C.A. Tesis de Maestría, Fac. de Ciencias, UNAM. México, D.F. 205p.

GONZÁLEZ-GONZÁLEZ, J. 1980. Some ecological considerations on the intertidal macroalgae of the Mexican and Central American tropical Pacific. Mem. II International Symposium on some tropical shallow water communities. Papua Nueva Guinea. 11p.

HOLLENBERG, G.J. 1944. An account of the species of Polysiphonia on the Pacific coast of North America. II. Polysiphonia. Amer. J. Bot. 31: 474-483.

HOLLENBERG, G.J. 1961. Marine red algae of Pacific Mexico. Part. 5. The genus Polysiphonia. Pacif. Nat. 2(6): 345-375.

HOLLENBERG, G.J. 1968. An account of the species of Polysiphonia of the central and western tropical Pacific Ocean. I. Oligosiphonia. Pacif. Sci. 22: 56-98.

HOLLENBERG, G. J. 1968 a. An account of the species of the red algae Polysiphonia of the central and western tropical Pacific Ocean. II. Polysiphonia. Pacif. Sci. 22: 198-207.

HOLLENBERG, G.J. y J.N. NORRIS. 1977. The red algae (Rhodomelaceae) in the northern Gulf of California. Smithsonian Contr. Marine Sci. 1: 21.

HOMMERSAND, M.H. 1963. The morphology and classification of some Ceramiaceae and Rhodomelaceae. Univ. Calif. Publ. Bot. 35:165-366.

JOLY,A.B. 1967. Gêneros de algas marinhas da costa Atlântica Latino-Americana. Editora da USP. Sao Paulo. 461 p.

KRAFT, G.T. 1981. Rhodophyta: Morphology and Classification. En: C.S. Lobban y M.J. Wynne (Edrs.) The Biology of Seaweed. Blacwell Scientific Pubs. London.pp: 6-51.

KYLIN, H. 1956. Die Gattungen der Rhodophyceen. XV + 673 CWK Cleerups, Lund.

MARTINELL, B.L. 1983. Estudio prospectivo de las algas rojas (Rhodophyta) de las desembocaduras dell Río Balsas. Tesis, Fac. de Ciencins, UNAM. México, D.F. 97p.

MARTINELL, B.L. 1986. Estudio ecológico de las algas de las desembocaduras de Michoacán. Tesis de Maestría. Fac. de Ciencias, UNAM. México, D.F. 179p.

OLIVEIRA-FILHO, C.E. 1969. Algas marihas do sul do estado do Espírito Santo (Brasil). I. Ceramiales. Botánica 26: 277.

PEDROCHE, F.F. y J.GONZÁLEZ-GONZÁLEZ. 1980. El proyecto macroalgas dell Pacífico mexicano y sus avances. II. Estrategia general de trabajo. Seminario USA-MEX. sobre los avances de la Ficología en ambos países. (mimeografiado) 11p. (de circulación limitada).

PEDROCHE, F.F.; Z. CORREA; K. DRECKMANN y A. SENTÍES. 1984. Prospección ficológica de las costas del estado de Miclıoacán, Méx. Resumenes 9 Congreso Mexicano de Botánica. Sociedad Botánica de México. pp. 279.

SAITO, Y. 1967. Studies on japanese species of Laurencia, with special reference to the comparative morphology. Mem. Fac. Fish Hokaido Univ., Jokadate. 15(1):1-81.

SAITO, Y. y B.S. WOMERSLEY. 1974. The southern Australian species of Laurencia (Ceramiales: Rhodophyta). Austr. J. Bot.22: 815-74.

SCAGEL, R.F. 1953. A morphological study of some dorsiventral Rhodomelaceae. Univ. Calif. Publ. Bot. 27: 1-108. 
SENTÍES, G.A. 1985. Estudio florístico preliminar de la Familia Rhodomelaceae (Ceramiales, Rhodophyta) en la costa del estado de Michoacán, Méx. Tesis, Fac. de Ciencias, UNAM. México, D.F. 66p.

SILVA, P.C. 1979. The benthic algal flora of central San Francisco Bay. En: Conomos, T.J. (Edr.) San Francisco Bay: The urbanized estuary. Pacific Division of the American Association for the Advancement of Science pp. 287-311.

TAYLOR, W. R. 1945. Pacific marine algae of the Allan Hancock Expedition to the Galapagos Islands. A. Hancock Pac. Exped. 12:1-528.

TAYLOR, W.R. 1960. Marine algae of the eastern tropical and subtropical coast of the Americas III. Ann Arbor University of Michigan Press. 870p.

TAYLOR, W.R. 1966. Variation in the genus Polysiphonia. Proceeding of the fifth international seaweed symposium Pergamon Press-Oxford. New York. 209-216p.

WOMERSLEY, H.B.S. 1979. Southern Australian species of Polysiphonia Greville (Rhodophyta). Austr. J. Bot. 27: 459- 528. 
TABLA 1. Registro de la presencia - ausencia de las especies en las localidades

TAXA:

LOCALIDADES:

AMBIENTES:

ST

$\mathbf{P}$

$\mathbf{M}$

Polysiphonia:

confusa

scopulorum

sphaerocarpa

$P$. sp.

Tayloriella:

dictyurus

Herposiphonia:

littoralis

plumula

tenella

Chondria:

arcuata

dasyphylla

decipiens

Laurencia:

clarionensis

hancockii

voragina
X

X

X

Prs, Rsc, Acn, Cen
Plr, Prs, Cen

Ccn

Prs, Plr, Cen, Rsc

Prs
Plr

Plr

Plr
Plr, Ccn, Rsc Plr, Ccn, Rsc

Plr

Clave: ST = San Telmo; $\mathrm{P}=$ Pichilinguillo; $\mathrm{M}=$ Mexcalhuacan; $\mathrm{x}=$ presencia; - = ausencia; Plr $=$ Plataforma rocosa; Prs = Punta rocosa $\mathrm{Ccn}=$ Canal de corriente; Rsc $=$ Risco; Acn = Acantilado. (Modificado de Sentíes, 1985 y Dreckmann, 1987). 